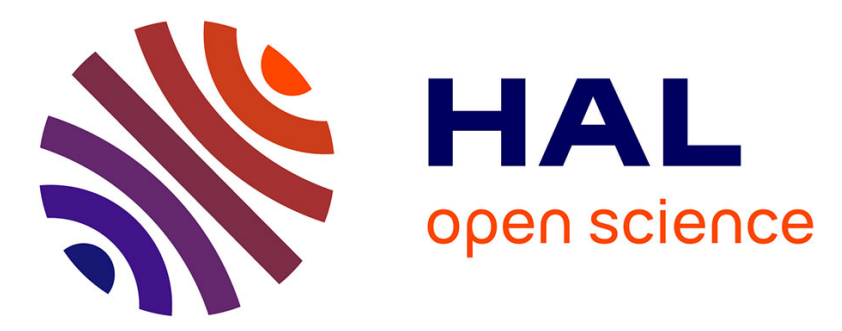

\title{
Magnetically induced nematic-smectic A tricritical point
}

C. Rosenblatt

\section{- To cite this version:}

C. Rosenblatt. Magnetically induced nematic-smectic A tricritical point. Journal de Physique Lettres, 1981, 42 (1), pp.9-12. 10.1051/jphyslet:019810042010900 . jpa-00231865

\section{HAL Id: jpa-00231865 https://hal.science/jpa-00231865}

Submitted on 1 Jan 1981

HAL is a multi-disciplinary open access archive for the deposit and dissemination of scientific research documents, whether they are published or not. The documents may come from teaching and research institutions in France or abroad, or from public or private research centers.
L'archive ouverte pluridisciplinaire HAL, est destinée au dépôt et à la diffusion de documents scientifiques de niveau recherche, publiés ou non, émanant des établissements d'enseignement et de recherche français ou étrangers, des laboratoires publics ou privés. 


\title{
Magnetically induced nematic-smectic A tricritical point
}

\author{
C. Rosenblatt (*) \\ Applied Laser Spectroscopy Group, Lawrence Berkeley Laboratory, University of California, Berkeley, California 94720, U.S.A.
}

(Reçule 22 août 1980, accepté le 6 novembre 1980)

\begin{abstract}
Résumé. - Il est montré théoriquement que, lors d'une transition nématique-smectique A, la présence d'un fort champ magnétique peut faire passer d'une transition du premier ordre à une transition du deuxième ordre. On considère ensuite un exemple spécifique basé sur le modèle de Ronis et Rosenblatt [Phys. Rev. A 21 (1980) 1687].
\end{abstract}

\begin{abstract}
It is shown theoretically that a strong magnetic field can induce a changeover from first to second order behaviour in the nematic-smectic A transition. A specific example is discussed based on the model of Ronis and Rosenblatt [Phys. Rev. A 21 (1980) 1687].
\end{abstract}

Magnetic fields have played a significant role in the field of liquid crystals. Due to the diamagnetic anisotropy of most liquid crystal molecules, the presence of fields as strong as a few kilogauss is generally sufficient to induce a uniform sample alignment [1]. In addition, magnetic fields have long been used to measure the elastic properties of liquid crystals by means of the Freedericksz transition.

More recently there has been a growing interest in the interaction of liquid crystals with very strong magnetic fields. Using Landau theory, Fan and Stephen [2] showed that above some critical field the nematic-isotropic (NI) transition can become second order if the diamagnetic anisotropy, $\chi_{a}$ is negative. Another interesting result was obtained by Wojtowicz and Sheng [3] in numerical calculations using the Maier-Saupe [4] model of the NI transition. Above some critical field $H_{\mathrm{c}}$ the paranematic (the isotropic phase with some small, magnetically-induced alignment) goes continuously into the nematic phase as the temperature is reduced ; in other words, a critical point occurs above which there is no discrete phase transition. Experimentally, however, such an effect would seem inaccessible in that $H_{c}$ was calculated to be of the order of $10 \mathrm{MG}$.

On the experimental side, Keyes and Shane [5] have used very strong magnetic fields and birefringent data to study the NI transition. Their results are consistent with the predictions of Keyes [6], namely,

(*) Present Address : Francis Bitter National Magnet Laboratory, Massachusetts Institute of Technology, Cambridge, Massachusetts 02139 , U.S.A. the NI transitions exponents exhibit tricritical rather than mean field behaviour. Finally, Poggi and Filippini [7] and Malraison, et al. [8] have studied the optical birefringence of nematics in the presence of intense fields. Noting that such fields tend to quench long-range collective fluctuations and increase the average value of the nematic order parameter $s$, they report that the induced birefringence varies linearly in field in the nematic phase ; this is in contrast to the quadratic Cotton-Mouton effect in the isotropic phase. Furthermore, the latter group reports that the induced birefringence is reduced near the nematicsmectic A (NS) transition because of the divergence of the bend elastic constant, and thus the correlation length, as the system goes smectic.

In this paper it is shown that a very strong magnetic field can change a first order NS transition into a second order transition. A simple Landau-De Gennes argument is given, and a specific material (identical to cholesteryl myristate in zero field) is considered in terms of the recent lattice model presented by Ronis and Rosenblatt [9, 10].

De Gennes [1] has shown that, in terms of a Landau free energy expansion, the coupling between the nematic and smectic order parameters can drive the NS transition first order. He wrote a free energy of the form :

$$
\begin{aligned}
F=\alpha_{0}|\Psi|^{2}+\beta_{0}|\Psi|^{4} & +F_{\mathrm{N}}\left(s_{0}\right)+ \\
& +\frac{1}{2 \chi} \delta s^{2}-C|\Psi|^{2} \delta s
\end{aligned}
$$

where $\chi$ is a susceptibility, the term containing $C$ couples the smectic order parameter $\Psi$ and the 
nematic parameter $s$, and where $s=s_{0}+\delta s . s_{0}$ is the orientational order parameter in the absence of smectic coupling or magnetic fields. After minimizing $F$ with respect to $\delta s$, he found that

$$
\delta s=\chi C \Psi^{2}
$$

and

$$
F=F_{\mathrm{N}}\left(s_{0}\right)+\alpha_{0} \Psi^{2}+\beta \Psi^{4}
$$

where $\beta$ is given by

$$
\beta=\beta_{0}-\frac{1}{2} C^{2} \chi
$$

When the nematic temperature range is small, $s$ hasn't yet saturated and $\chi$ is large. Consequently $\beta$ is negative and the transition is first order, consistent with experiment. On the other hand, for small $\chi$ the transition is second order.

To include magnetic field effects we add a term $F_{\mathrm{M}}=-K H^{2} \delta s$ to equation (1). $F_{\mathrm{M}}$, expanded to lowest order in $H$, involves both the entropy and internal energy contributions to $F$; the positive constant $K$ is proportional to the diamagnetic anisotropy $\chi_{\mathrm{a}}\left[\equiv\left(\chi_{\|}-\chi_{\perp}\right)\right]$. (Note that $\chi_{\mathrm{a}}$ used here is the saturated diamagnetic anisotropy with orientational order $s=1$. In other words, $\chi_{\mathrm{a}}=N \alpha_{\mathrm{a}}$, where $N$ is the number density and $\alpha_{\mathrm{a}}$ the molecular diamagnetic anisotropy). After minimizing $F^{\prime}$ with respect to $\delta s$, we obtain

$$
\delta s=\chi\left(C \Psi^{2}+K H^{2}\right) .
$$

$H$ alone increases $s$ over $s_{0}$, as expected. Furthermore,

$$
F^{\prime}=F_{\mathrm{N}}\left(s_{0}\right)-\frac{1}{2} K^{2} H^{4} \chi+\alpha^{\prime} \Psi^{2}+\beta^{\prime} \Psi^{4}
$$

where

$$
\begin{aligned}
& \alpha^{\prime}=\alpha_{0}-C \chi K H^{2} \\
& \beta^{\prime}=\beta_{0}-\frac{1}{2} C^{2} \chi .
\end{aligned}
$$

The magnetic field increases the transition temperature directly through equation (7). Moreover, since a large field quenches microscopic fluctuations and thereby reduces $\chi, H$ affects the order of the transition indirectly through equation (8). Thus a sufficiently large field can drive the NS transition second order.

To obtain numerical results the lattice model of Ronis and Rosenblatt $[9,10]$ is used. Difficulties with a limited Fourier representation inherent in other models are not encountered in this model, and the phase diagram (Fig. 2, Ref. [9]) quite closely approximates real behaviour. In the model the parameter $R$ is physically related to the inverse of molecular length and $R^{\prime}$ serves as a coupling constant between nematic and smectic order parameters. Both parameters can be obtained by expanding the intermolecular pair potential or, more practically, by fitting the model to experimental data such as transition temperatures and entropies.

In the presence of a magnetic field an additional term in the new internal energy $U^{\prime}$ appears [1] :

$$
\begin{aligned}
U_{\mathrm{M}} & =-\sum_{i} \frac{1}{2} \chi_{\mathrm{a}} \Lambda\left(\mathbf{H} . \mathbf{\Omega}_{i}\right)^{2} \\
& =-\sum_{i} \frac{1}{3} \chi_{\mathrm{a}} \Lambda H^{2} s_{i}+\text { constant }
\end{aligned}
$$

$\Lambda$ is the volume of a lattice cell $\left[=(d / 2)^{3}\right.$, where $d$ is the smectic A layer spacing], $\boldsymbol{\Omega}$ is the director, and $s_{i}$ is $\left\langle n_{i} P_{2}\left(\cos \theta_{i}\right)\right\rangle$.

The mean fields $\mathcal{H}_{i}$ (Eq. (2.16), Ref. [9]) must be modified by terms of this sort and become

$$
\mathscr{H}_{i}^{\prime}=\mathscr{H}_{i}-\frac{1}{3} \chi_{\mathrm{a}} \Lambda H^{2} .
$$

Proceeding as in reference [9] it is easy to show that the new entropy is of the form :

$$
-T S^{\prime}=-T S+\sum_{i} \frac{1}{3} \chi_{\mathrm{a}} \Lambda H^{2} s_{i}
$$

Note that $S$ implicitly contains $H^{2}$ through the logarithm of the partition function. The explicit summation terms (Eqs. (9) and (10)) cancel in the new Helmholtz potential $A^{\prime}$; however, $H^{2}$ does enter $A^{\prime}$, again through the partition function.

We now consider a specific example such that $T_{\mathrm{NS}} / T_{\mathrm{NI}}=0.986$ and the NS transition entropy is $\delta S / k_{\mathrm{b}}=0.43$. ( $T_{\mathrm{NS}}$ is the nematic-smectic transition temperature and $T_{\mathrm{NI}}$ is the isotropic-nematic temperature.) Upon fitting these typical values to the model, we find material parameters $R=0.79$ and $R^{\prime}=0.43$. Moreover, we set $\Lambda=5.5 \times 10^{-22} \mathrm{~cm}^{3}$ and $\chi_{\mathrm{a}}=1.3 \times 10^{-7}$, where $\chi_{\mathrm{a}}$ is a value typical of many materials [1]. [Note that the material parameters $R$ and $R^{\prime}$ correspond identically to cholesteryl myristate, and therefore the temperature behaviour at zero field is shown in figure 3 of reference [9]. Cholesteryl myristate, however, has a negative diamagnetic anisotropy [11]; for the above material, on the other hand, we assume $\chi_{\mathrm{a}}>0$. Positive values of $\chi_{\mathrm{a}}$ are far more common [12].] The parameter $\Delta$ as shown in figure 3 of reference [9] is the strength of smectic ordering and defined as the difference in occupation $\left\langle n_{1}\right\rangle-\left\langle n_{2}\right\rangle$ between adjacent cells, such that the sum of the average occupations is equal to one. (See Eq. (3.2), Ref. [9].) $\Delta$ varies as a function of temperature between zero (nematic) and one (saturated smectic A). Since this material is first order at zero field, $\Delta$ jumps discontinuously from 0.0 to $0.34(\delta \Delta=0.34)$ at the NS transition. Now, as the magnetic field is increased, the discontinuity $\delta \Delta$ in going from nematic to smectic decreases from the zero field case, and is shown in figure $1 a$. At a field of $H_{\mathrm{T}}=1.8 \times 10^{7} \mathrm{G}$, the transition reaches a tricritical point. (The question of the large magnitude of the field will be addressed later.) Here the discontinuity $\delta \Delta$ goes to zero and, above $H_{\mathrm{T}}$, the transition is second order. (As the field is increased, a small increase in the nematic order parameter $s$ is noted (see Fig. 1c). Thus, at high fields the susceptibility is reduced and, from De Gennes argument (cf. Eqs. (6) and (8)), it is clear that the transition becomes 


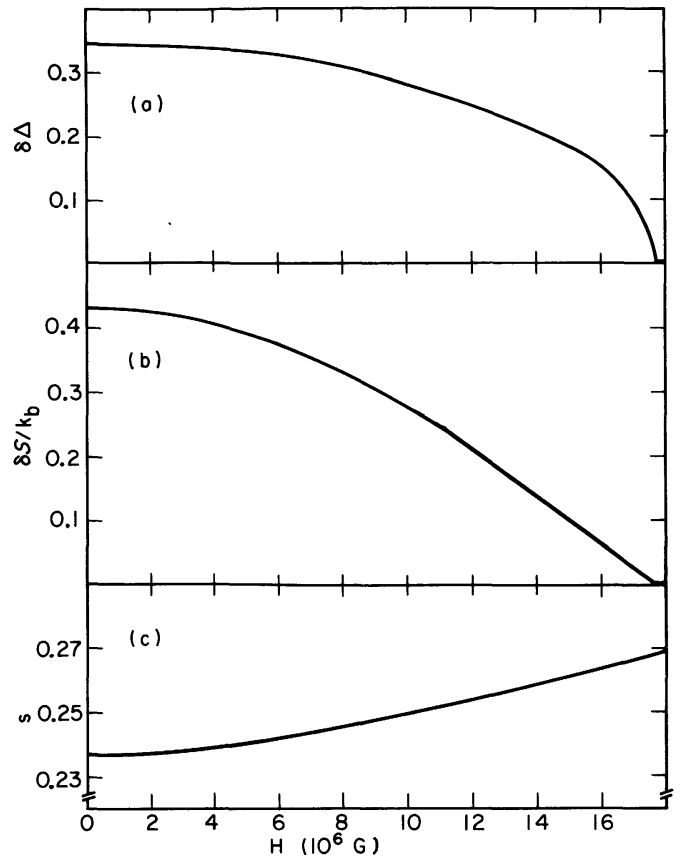

Fig. 1. - Behaviour of material with parameters $R=0.79$ and $R^{\prime}=0.43$. a) Discontinuity in the smectic order parameter $\delta \Delta$ at the NS transition vs. $H$. b) Discontinuity in the entropy $/ k_{\mathrm{b}}$ of transition $v s . H . c)$ Order parameter $s_{i}$ in the nematic phase at the transition temperature $T_{\mathrm{NS}}(H)$.

second order for sufficiently small $\chi$.) In figure $1 b$ the discontinuity in entropy $\delta S / k_{\mathrm{b}}$ is shown and, analogous to $\delta \Delta$, this quantity also vanishes at $H_{\mathrm{T}}$.

To determine the behaviour of $\delta \Delta$ and $\delta S / k_{\mathrm{b}}$ just below $H_{\mathrm{T}}$, we define $\delta H=H_{\mathrm{T}}-H$ and, assuming $\delta H$ is an analytic function of $\delta \Delta$, expand $\delta H$ in powers of $\delta \Delta$. According to the model [9], states of $+\Delta$ and $-\Delta$ are equivalent; thus $\delta H \propto \delta \Delta^{2}$ or, equivalently, $\delta \Delta \propto(\delta H)^{1 / 2}$. This behaviour is seen in figure $1 a$. Using a similar argument, it can be shown that $\delta S / k_{\mathrm{b}} \propto \delta H$.

In addition to finding decreased discontinuities in the order parameters, we also find that $T_{\mathrm{NS}}$ is an increasing function of $H$. Since an intense magnetic field saturates the orientational order $s$, one would expect the transition to occur at higher temperatures. Furthermore, $T_{\mathrm{R}}\left[\equiv\left(T_{\mathrm{NS}}(H)-T_{\mathrm{NS}}(0)\right) / T_{\mathrm{NS}}(0)\right]$ must be linear in $H^{2}$ (for sufficiently small fields) by symmetry; this behaviour is shown in figure 2. (We note in passing that Sakamoto, et al. [13] observed decreasing $T_{\mathrm{Ns}} v s . H$ behaviour at very small fields $\overline{(500 \mathrm{G}) \text {. }}$ Their theoretical explanation, however, fails to consider the effects of $H$ on entropy. Nevertheless, we are at a loss to explain both the large magnitude and sign of their experimental results. Reference [8], on the other hand, reports no observable shift in $T_{\mathrm{Ns}}(H)$ up to fields of $H=120 \mathrm{kG}$.)

Although the possible change from a first to a second order transition in the presence of a magnetic field is exciting, two problems exist with this calculation. The first is the very high value of $H_{\mathrm{T}}$, rendering

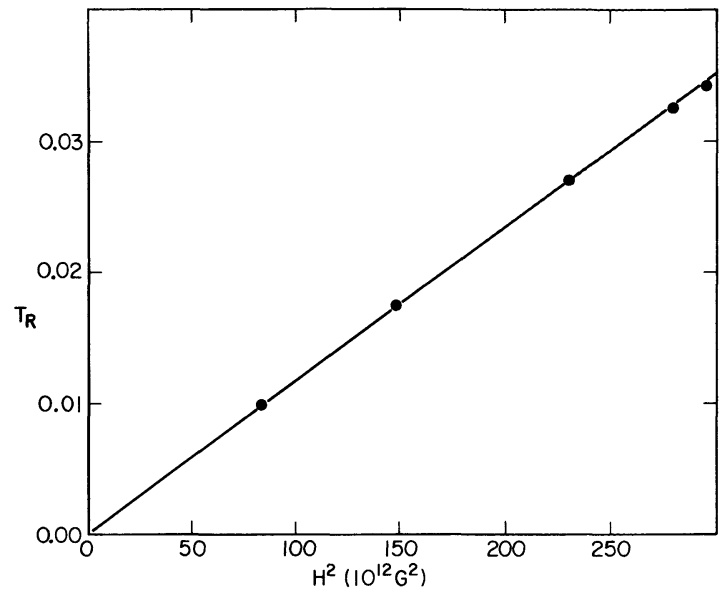

Fig. 2. - Reduced temperature

$$
T_{\mathrm{R}}\left[\equiv\left(T_{\mathrm{NS}}(H)-T_{\mathrm{NS}}(0)\right) / T_{\mathrm{NS}}(0)\right] v s . H^{2} .
$$

Five points, calculated using the model in reference [9], are shown. Notice the temperature increase deviates slightly from linearity at high fields.

experimental verification with this material quite unlikely. Another sample with material parameters $R=1.00$ and $R^{\prime}=0.50 \quad$ (corresponding to $T_{\mathrm{NS}} / T_{\mathrm{NI}}=0.946$ and $\left.\delta S / k_{\mathrm{b}}=0.02\right)$ was studied. Despite the fact that this material exhibits very weak first order behaviour, $H_{\mathrm{T}}$ is nevertheless about $4 \times 10^{6} \mathrm{G}$. (Qualitatively this material behaves similarly to figures 1 and 2.) To obtain magnetically induced tricritical behaviour one would undoubtedly have to use even weaker first order materials. Such materials can be manufactured using a binary mixture, as has been done by the Kent State Group $[14,15]$ and by Achard, et al. [16]. At this time, however, it is still uncertain whether reaching a tricritical point is experimentally possible. (We note that a first order material inherently close to the tricritical point (such as $R=1.00 / R^{\prime}=0.50$ above), exhibits a large susceptibility in the smectic order parameter $\Delta$. Thus, just below $T_{\mathrm{NS}}$, a field of $200 \mathrm{kG}$ can induce a change in $\Delta$ by as much as $10 \%$. Thus, although a tricritical point may be difficult to see, various trends may be experimentally accessible; in this case, an $\mathrm{X}$-ray study [17] of $\Delta$ may yield useful information.)

The second problem is inherently in the model. To date, no numerical model of the NS transition accounts for long wavelength collective fluctuations, as those studied in references [7] and [8]. There it was determined that the nematic induced birefringence is collective in nature and exhibits linear behaviour in $H$; it is unaffected in the smectic phase because of the diverging correlation length $\xi=\left(K_{33} / \chi_{\mathrm{a}} H^{2}\right)^{1 / 2}$. On the nematic side of the transition, therefore, the low field $\left(H \ll H_{\mathrm{T}}\right)$ behaviour will be dominated by collective effects, and many of the nematic calculations presented in this paper may be in error. (The smectic results should be far better because of the divergence of $K_{22}$ and $K_{33}$.) On the other hand, 
at very high fields (near $H_{\mathrm{T}}$ ), the correlation length will have become quite small (tens of angströms) and, sufficiently far from $T_{\mathrm{NS}}(H)$ (such that the elastic constants haven't yet exhibited divergent behaviour), the nematic calculations should be relatively good.

Halperin and Lubensky [18] and Halperin, Lubensky, and Ma [19] have, in addition, shown that long wavelength fluctuations will always drive the NS transition weakly first order. However, they also recognized [18] that a sufficiently strong magnetic field will restore the second order behaviour of the transition by quenching the small $\mathbf{q}$ fluctuations. In a type I superconductor (as most smectics should behave $[20,21])$, the second term in equation (8) of reference [19] is linear in $k_{\mathrm{s}}$, the inverse of the London penetration depth; this term is responsible for the first order behaviour. However, the presence of a field (in the liquid crystal analogy) effectively adds a term proportional to $H^{2}$ to the free energy expansion, thus inhibiting long wavelength modes. For $H$ sufficiently large, the second term in equation (8) of reference [19] will then go as $k_{\mathrm{s}}^{2} / H$, rather than $k_{\mathrm{s}}$. Thus, the cubic term in the free energy $F$ (the first integral of Eq. (5), Ref. [19]) vanishes and the transition reverts to second order. Therefore, for sufficiently large fields (yet still well below $H_{\mathrm{T}}$ ), the long wavelength fluctuations will not necessarily induce first order behaviour, and the NS transition can exhibit a real tricritical point.

In this paper we conjecture that a sufficiently strong magnetic field can induce NS tricritical behaviour. Despite the difficulties with long wavelength fluctuations (on the nematic side) and the large critical fields, experiments are being planned to study various pretransitional trends.

Acknowledgments. - The author wishes to thank Ronald Pindak of Bell Labs. and Robert Meyer of Brandeis for helpful comments. In addition, the comments by and discussions with David Ronis of Harvard and Nabil Amer of the Lawrence Berkeley Laboratory are particularly appreciated. This work was supported by an NSF contract to the Francis Bitter National Magnet Laboratory.

\section{References}

[1] See, for example, De Gennes, P. G., Physics of Liquid Crystals (Clarendon, Oxford) 1975.

[2] Fan, C.-P. and Stephen, M. J., Phys. Rev. Lett. 25 (1970) 500.

[3] Wojtowicz, P. J. and Sheng, P., Phys. Lett. 48 A (1974) 235.

[4] MAIER, W. and SAUPE, A., Z. Naturforsch. 13A (1958) 564 ; 14A (1959) $882 ;$ 15A (1960) 287.

[5] Keyes, P. H. and Shane, J. R., Phys. Rev. Lett. 42 (1979) 722.

[6] Keyes, P. H., Phys. Lett. 67A (1978) 132.

[7] Poggi, Y. and Filippini, J. C., Phys. Rev. Lett. 39 (1977) 150.

[8] Malraison, B., Poggi, Y. and Guyon, E., Phys. Rev. A 21 (1980) 1012.

[9] Ronis, D. and Rosenblatt, C., Phys. Rev. A 21 (1980) 1687

[10] Rosenblatt, C. and Ronis, D., Phys. Rev. A 23 (1981) 305.

[11] Sackmann, E., Meiboom, S., Snyder, L. C., Meixner, A. E. and DieTz, R. E., J. Am. Chem. Soc. 90 (1968) 3567.

[12] The author knows of no first order NS material for which both the entropy of transition and the saturated diamagnetic susceptibility are known. Hence, this very typical example was chosen.
[13] SaKamoto, A., Yoshino, K., Kubo, U. and InUishi, Y., Japan. J. Appl. Phys. 15 (1976) 545.

[14] Johnson, D. L., Maze, C., Oppenheim, E. and Reynolds, R., Phys. Rev. Lett. 34 (1975) 1143.

[15] Brisbin, D., Dehoff, R., Lockhart, T. E. and Johnson, D. L., Phys. Rev. Lett. 43 (1979) 1171.

[16] Achard, M. F., Hardouin, F., Sigaud, G. and GaspaRoux, H., J. Chem. Phys. 65 (1976) 1387.

[17] Stamatoff, J., Cladis, P., Guillon, D., Cross, M., Bilash, T. and FinN, P., Phys. Rev. Lett. 44 (1980) 1509.

[18] Halperin, B. I. and Lubensky, T. C., Solid State Commun. 14 (1974) 997.

[19] Halperin, B. I., Lubensky, T. C. and Ma, S.-K., Phys. Rev. Lett. 32 (1974) 292.

[20] De Gennes, P. G., Solid State Commun. 10 (1972) 753.

[21] De Gennes, P. G., Mol. Cryst. Liq. Cryst. 21 (1973) 49. 